\title{
Rational Engineering of BODIPY-Bridged Trisindole Derivatives for Solar Cell Applications
}

\author{
Ibrahim Bulut ${ }^{+}{ }^{[a]}$ Quentin Huaulmé ${ }^{+[a]}$ Antoine Mirloup, ${ }^{[\mathrm{a}]}$ Patricia Chávez, ${ }^{[\mathrm{a}]}$ Sadiara Fall, ${ }^{[\mathrm{b}]}$ \\ Anne Hébraud, ${ }^{[a]}$ Stéphane Méry, ${ }^{[c]}$ Benoît Heinrich, ${ }^{[c]}$ Thomas Heiser, ${ }^{[b]}$ Patrick Lévêque, ${ }^{*[b]}$ \\ and Nicolas Leclerc*[a]
}

\begin{abstract}
academic titles added for Dr. Lévêque and Dr. Leclerc; ok? Boron dipyrromethene (BODIPY) and its derivatives are known to be efficient photon-harvesting chromophores. However, their study as active materials in bulk heterojunction $(\mathrm{BHJ})$ solar cells is still scarce. In this study, the development of new synthetic ways to design original BODIPY-based dumbbell-shape molecules, including a first 2,3,5,6-tetravinyl aromatic BODIPY molecule, is reported. High fill factors can be obtained in $\mathrm{BHJ}$ solar cells when blended with a fullerene derivative, leading to a new record BODIPY-based power conversion efficiency of $5.8 \%$.
\end{abstract}

Boron dipyrromethene (BODIPY) chromophores are well known for their remarkably high photon extinction coefficients, excellent photostability and relative ease of chemical functionalization, that led to their use in numerous applications. ${ }^{[1]}$ BODIPY derivatives have been designed to achieve specific frontier orbital energy levels or tailored photon absorption/ emission spectra. ${ }^{[2]}$ These features are particularly important for organic photovoltaic (OPV) devices, as demonstrated recently by Bura et al., ${ }^{[3]}$ where the BODIPY core has been functionalized at the 3 and 5 positions with bithiophene units to extend the conjugation length and to optimize the frontier orbital energy levels. In that study, devices based on a BODIPY derivative $\left(\mathrm{TB}_{2}\right)$ blended with [6,6]-phenyl- $\mathrm{C}_{61}$-butyric acid methyl ester $\left(\mathrm{PC}_{61} \mathrm{BM}\right) \mathbf{\square}$ ok? led to a power conversion efficiency (PCE) close to $5 \%$, whereas the expected achievable PCE was estimated as $10 \% .{ }^{[4]}$ The modest fill factor (FF) was the major efficiency-limiting factor. FFs of OPV devices depend to a large extent on the charge carrier mobility and therefore on the strength of intermolecular interactions and on structur-

[a] Dr. I. Bulut, ${ }^{+}$Dr. Q. Huaulmé, ${ }^{+}$Dr. A. Mirloup, Dr. P. Chávez, Dr. A. Hébraud, Dr. N. Leclerc

ICPEES UMR 7515, Université de Strasbourg-CNRS

25 rue Becquerel, 67087 Strasbourg (France)

E-mail: leclercn@unistra.fr

[b] Dr. S. Fall, Prof. T. Heiser, Dr. P. Lévêque

ICube UMR 7357, Université de Strasbourg-CNRS

23 rue du Loess, 67037 Strasbourg (France)

E-mail: patrick.leveque@unistra.fr

[c] Dr. S. Méry, B. Heinrich

IPCMS UMR 7504, Université de Strasbourg-CNRS

23 rue du Loess, 67034 Strasbourg (France)

$\left.{ }^{[}\right]$These authors contributed equally to this work.

$\square$ Supporting information for this article can be found under: http://dx.doi.org/10.1002/cssc.201700465. al order. Enhancing molecular interactions in conjugated materials may be addressed by introducing specific functional groups or by tuning the solubilizing side-chains and could potentially lead to higher performances. An example is given by the seminal work of Lee et al., who added planar conjugated moieties on opposite sides of molecular dyes to promote molecular stacking. ${ }^{[5]}$ More recently, the utilization of triazatruxene (TAT) derivatives as planar end groups grafted onto different molecular dyes was reported by Bulut et al. ${ }^{[6]}$ only 6 is Bulut et al. what about $6 \mathrm{~b} ?$ Their results suggested that the TAT-dye-TAT architecture led to strengthened molecular stacking behavior, while the optical properties were still essentially determined by the central dye. In this study, we follow the same strategy to positively alter the self-assembling properties of the $\mathrm{TB}_{2}$ derivative and explore its consequences on OPV device performances.

The TAT units can in principle be grafted at the available 2 and 6 positions of the $\mathrm{TB}_{2}$ core (Figure 1). However, Lin et al. reported that direct carbon-carbon coupling at these positions introduces steric hindrance that weakens the electronic conjugation and bends the molecule. ${ }^{[7]}$ The insertion of an alkyne linker was found to release the steric congestion and resulted in improved light-harvesting, charge-transport, and, finally, photovoltaic performances. Taking into account the fact that $\mathrm{TB}_{2}$ is functionalized by bulky side groups (methyl groups at 1 and 7 positions and vinylbithiophene moieties at 3 and 5 positions) and trying to avoid large twist angles, we decided to link the sterically demanding TAT fragments to the BODIPY core via a conjugated linker. We used for this purpose either ethynylene (BET in Figure 1) or vinylene (BVT in Figure 1), the latter being known to favor conjugation extension in its $E$ (trans) configuration. ${ }^{[8]}$ Herein, we describe the challenging synthesis of both TAT-TB 2 -TAT molecules, as well as their optoelectronical properties. Finally, we have demonstrated that the BET derivative yields a significant increase in PCE over that of $\mathrm{TB}_{2}$, which we attribute to better charge collection resulting from the enhanced intermolecular interactions driven by the TAT end groups.

The synthetic routes for the preparation of BVT and BET molecules (Scheme 1) require the preparation of the 2,6diiodo- $\mathrm{TB}_{2}$ derivative 4 (see the Supporting Information). The ethynyl BET compound was synthesized by a standard Sonogashira reaction between $\mathbf{4}$ and the ethynyl-TAT derivative $\mathbf{2}$ and isolated in $50 \%$ yield. Regarding the vinyl BVT compound, the synthesis is more complex. Indeed, BODIPY dyes carrying four vinylaryl moieties at $3,5(\alpha), 2$, and $6(\beta)$ positions have, to our 


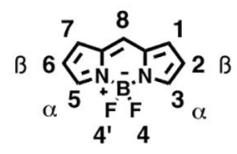

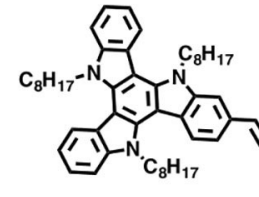
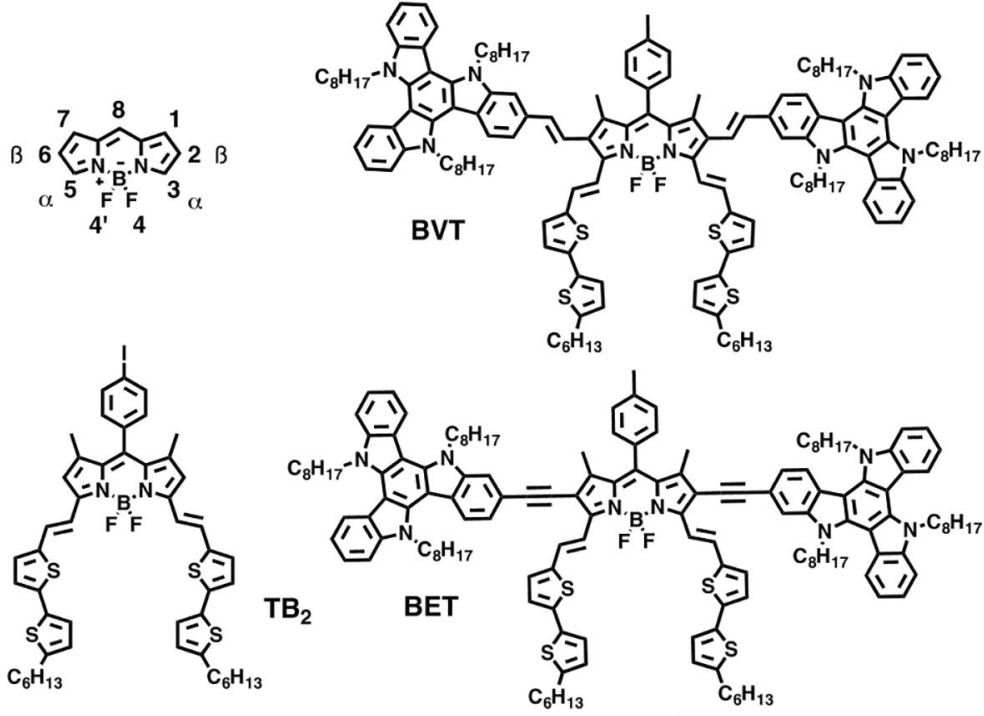<smiles>c1ccccc1</smiles>

Figure 1. Numbering scheme used for the BODIPY framework (top left), initial $\mathrm{TB}_{2}$ molecule, and new dumbbell-shaped BVT and BET molecules.

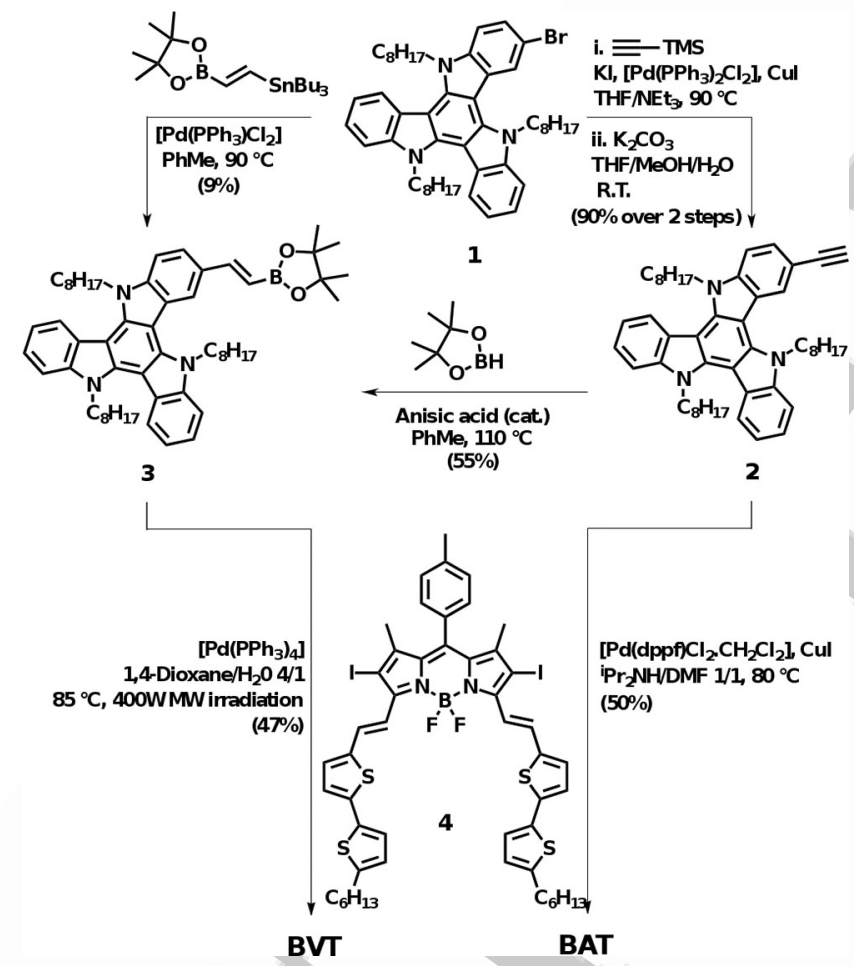

Scheme 1. Synthetic routes for the preparation of the BVT and BET molecules.

knowledge, not been reported to date and therefore require the development of a specific synthesis protocol. BODIPY dyes with vinyl groups at 2 and 6 positions were reported very recently, but only with methyl groups at the 3 and 5 positions. ${ }^{[9,10]}$ Bröring and co-workers synthesized a BODIPY dimer, composed of two BODIPY cores linked together at the $\beta$ positions by a vinyl bond, by using a methatetic homodimerization approach. ${ }^{[9]}$ Unfortunately, dimerization of the 1,3,5,7-tetra- methyl hindered BODIPY core following metathesis reaction optimization with the highly active Grubbs II catalyst could only be achieved with a low yield (17\%). The poor efficiency of this reaction associated to its limited access to heterocouplings, led us to consider other synthetic pathways. Shen and coworkers almost simultaneously demonstrated that the 2,6-distyryl BODIPY could be synthesized through a Heck reaction from a 2,6-diiodo-BODIPY derivative. ${ }^{[10]}$ However, when using an electron-rich p-dimethylaminostyryl building block, the authors observed the formation of three isomers with styryl substituents connected either at the $\beta$ or $\alpha$ positions. Because such uncontrolled isomerization leads to only a $9 \%$ yield of the isolated targeted $\beta$-substituted $\square$ ok? isomer, this Heck-approach was not retained either. We also note the recent first example of a BODIPY substituted by four vinyl esters obtained by Heck coupling. ${ }^{[11]}$ However, this compound displays four identical non-aromatic methyl acrylate residues in at the 2, 3, 5, and 6 positions. Vinylaryl moieties are usually introduced on BODIPY cores by Knœvenagel-type condensation of methyl groups. Although this strategy is rather efficient for methyl groups at the 1, 3, 5, and 7 positions, Akkaya and co-workers demonstrated that the methyl groups at the 2 and 6 positions are not nucleophilic enough to react with aldehydes, leading to the decomposition of the dye instead of the formation of the expected vinyl adduct. ${ }^{[12]}$ Among the remaining possibilities to insert vinylaryl moieties are the Horner-Wadsworth-Emmons (HWE) and Wittig series of reactions. Unforerence? Unately, owing to the general instability of the BODIPY unit under basic conditions, the condensation of the phosphoranyl reactive groups on the 2,6-dicarbaldehyde-BODIPY unit led in both cases to its almost complete degradation.

Herein, we show that it is possible to insert with high yields vinylaryl moieties at the 2 and 6 positions of the BODIPY core by using the vinyldioxaborolane TAT derivative $\mathbf{3}$ via a SuzukiMiyaura cross-coupling reaction. Compound $\mathbf{3}$ can in principle be synthesized in one step, from the commercially available 4,4,5,5-tetramethyl-2-[(1E)-2-(tributylstannyl)ethenyl]-1,3,2-dioxaborolane, but this reaction proceeds at a very low yield (7\%). An interesting alternative to produce 3 in a much more efficient way is possible by hydroboration of the pivotal derivative 2 using 4,4,5,5-tetramethyl-1,3,2-dioxaborolane (Scheme 1). Following this approach, we obtained compound 3 in its trans configuration exclusively, isolated in $55 \%$ yield. A subsequent double Suzuki cross-coupling of $\mathbf{3}$ with $\mathbf{4}$ finally afforded the bis(vinylaryl) derivative BVT, isolated in $47 \%$ yield. This BVT compound constitutes the first example of tetra(vinylaryl)-substituted BODIPY molecule with different vinylaryl groups at the 3,5 and 2,6 positions. Both BVT and BET were characterized by NMR spectroscopy and MALDI-TOF mass spectrometry $\mathbf{\square}$ ok? There is no mention of MALDI in the Supporting Information

(see the Supporting Information). The appearanceof the four doublets of the vinylene linkers, exhibiting characteristic 
coupling constants of approximately $16 \mathrm{~Hz}$, confirms the BVT trans stereoselectivity.

UV/Vis absorption spectra of dilute chloroform solutions and thin films of BET, BVT, and the reference compound $\mathrm{TB}_{2}$ are shown in Figure 2. The corresponding spectroscopic data are
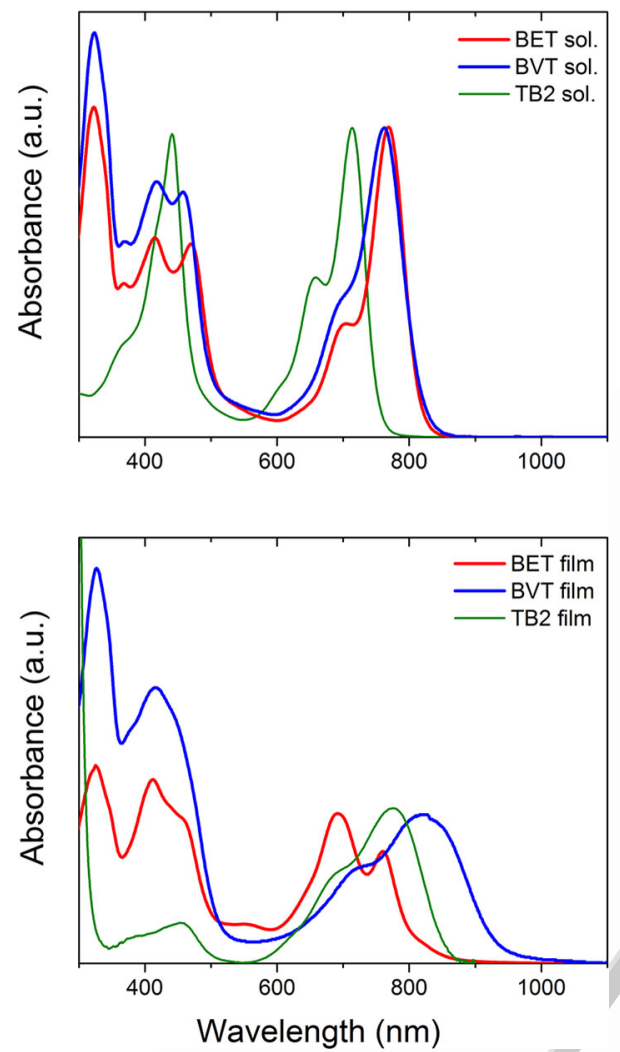

Figure 2. Normalized absorption spectra for $\mathrm{TB}_{2}$, BET, and BVT in solution (top) and in film (bottom).

gathered in Table S1. In solution, both BET and BVT dyes exhibit an optical band gap of $1.5 \mathrm{eV}$. The approximate $50 \mathrm{~nm}$ bathochromic shift of the low-energy absorption peak with respect to $\mathrm{TB}_{2}$ indicates that the TAT units extend slightly the conjugation length. These results are in line with reported data where bathochromic shifts of about $40-60 \mathrm{~nm}$ have been reported for BODIPY derivatives with conjugation extension at positions 2 and $6 .^{[13]}$ For both molecules, the optical band gap is in accordance with their electrochemical gap (1.35 eV) measured by cyclic voltammetry (see the Supporting Information). The thin film absorption spectra of BVT and BET molecules differ significantly (Figure 2). The BVT spectrum shows a broadening of the low-energy peak with a large bathochromic shift $(95 \mathrm{~nm})$, leading to a low optical band gap of about $1.3 \mathrm{eV}$. In contrast, the BET thin film spectrum reveals the appearance of a new intense absorption band at $690 \mathrm{~nm}$, which is most likely reflecting the formation of $\mathrm{H}$-type aggregates, as found for other BODIPY dyes with head-to-tail organization. ${ }^{[14]}$ The absorption onset at about $815 \mathrm{~nm}$ corresponds to an optical band gap of $1.52 \mathrm{eV}$, slightly above the value for $\mathrm{TB}_{2}(1.45 \mathrm{eV})$.
The differences in film absorption spectra suggest that the solid-state packing of BET and BVT differs significantly. DFT calculations of the molecular conformations of BET and BVT show that the vinyl linker induces a large torsion angle (around $35^{\circ}$ ) between the BODIPY core and the TAT units, whereas for the ethynyl linker the angle is only about $12^{\circ}$ (see the Supporting Information). As a consequence, both TAT end groups are expected be almost coplanar for BET, which is a priori favorable for $\pi$-stacking among neighboring TAT units, whereas those of BVT should be twisted and therefore more conducive to inducing structural disorder. Since the frontier orbitals are mainly localized on the BODIPY core, these conformational differences are not expected to have a strong impact on the HOMO and LUMO levels in solution, but they may change considerably the solid-state packing and the corresponding phase transitions. The latter properties were investigated by using polarized optical microscopy and differential scanning calorimetry (see the Supporting Information). The birefringence observed for the as-deposited thin films suggests that both materials are in the crystalline state, as confirmed by the melting temperatures of $170^{\circ} \mathrm{C}$ and $230^{\circ} \mathrm{C}$ found in the bulk state for BVT and $\mathrm{BET}$, respectively. The significantly lower melting temperature of BVT indicates weaker intermolecular coupling and corroborates the impact of the molecular conformation on the molecular packing.

The charge transport properties of both materials were probed in organic field-effect transistors. The corresponding field-effect hole mobilities were found to be of the same order of magnitude for BVT $\left(2.7 \times 10^{-4} \mathrm{~cm}^{2} \mathrm{~V}^{-1} \mathrm{~s}^{-1}\right)$ and BET $(5.4 \times$ $\left.10^{-4} \mathrm{~cm}^{2} \mathrm{~V}^{-1} \mathrm{~s}^{-1}\right)$ and were slightly lower than that reported for $\mathrm{TB}_{2}\left(10^{-3} \mathrm{~cm}^{2} \mathrm{~V}^{-1} \mathrm{~s}^{-1}\right)$. At this stage, we considered that the mobilities were sufficiently high to use both molecules as donor materials in BHJ solar cells. Accordingly, both dyes were tested as electron-donor species in blends with $[6,6]$-phenyl- $C_{71}$-butyric acid methyl ester $\left(\mathrm{PC}_{71} \mathrm{BM}\right) \mathbf{\square}$ ok? $\mathbf{\square}$, using an inverted device structure. The optimum device performances (Figure $3 \mathrm{a}$ ) are summarized in Table 1, together with the previously reported results for devices based on $\mathrm{TB}_{2}$. Large differences in the optimum elaboration conditions (see the Supporting Information) were found for both molecules. These dissimilarities are likely a consequence of the differing molecular assemblies of BET and BVT mentioned above and of the correspondingly different morphologies of the donor and acceptor domains.

BET gave the highest PCE value of $5.8 \%$, which is well above that for the best-performing $\mathrm{TB}_{2}$ device. Interestingly, this increase in performance is due to a much higher FF $(65 \%$ instead of $47 \%$ ). In contrast, BVT gave much lower performances despite a better photon-harvesting ability (lower band gap). In this case, both the FF and photocurrent dropped drastically, indicating deficient charge generation and collection. Many physical mechanisms may be at the origin of these very large discrepancies. Since a full investigation of the loss mechanisms for both molecules lies beyond the scope of this study, we herein focus only on the transport and recombination kinetics of BET and its comparison with $\mathrm{TB}_{2}$.

Additional out-of-plane hole transport measurements were performed on BET and BET:PC ${ }_{71} \mathrm{BM}$ thin films, as well as on $\mathrm{TB}_{2}$ 

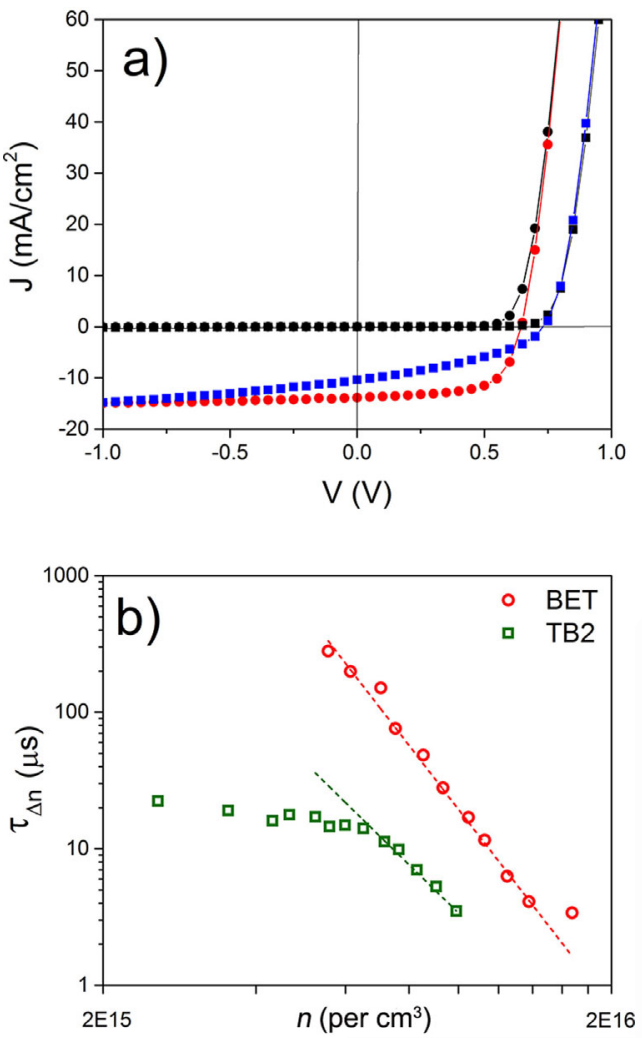

Figure 3. a) $J-V$ characteristics in the dark (black symbols) and under illumination (colored symbols) for solar cells based on BET (circles) and BVT (squares. b) Excess charge-carrier recombination time $\left(\tau_{\Delta n}\right)$ as a function of the charge carrier concentration ( $n$ ) measured on $\mathrm{BET}: \mathrm{PC} \mathrm{C}_{71} \mathrm{BM}$ and $\mathrm{TB} 2: \mathrm{PC}_{61} \mathrm{BM}$ solar cells using a combination of TPV and CE techniques.

Table 1. Maximum photovoltaic parameters obtained with BET and BVT, compared to $\mathrm{TB}_{2}{ }^{\left[{ }^{[3]}\right.}$

\begin{tabular}{|lllll|} 
Molecule & $V_{\text {oc }}[\mathrm{V}]$ & $J_{\mathrm{sc}}\left[\mathrm{mAcm}^{-2}\right]$ & $\mathrm{FF}[\%]$ & PCE [\%] \\
\hline BET & $0.64(0.65)^{[\mathrm{a}]}$ & $13.9(12.8)$ & $65(63)$ & $\mathbf{5 . 8}(5.3)$ \\
BVT & $0.75(0.74)$ & $10.0(10.4)$ & $39(38)$ & $2.9(2.9)$ \\
TB $_{2}$ & 0.70 & 14.3 & 47 & 4.7 \\
\hline
\end{tabular}

[a] Values in parentheses are average values from 20 different diodes.

and $\mathrm{TB}_{2}: \mathrm{PC}_{61} \mathrm{BM}$ blends $\left(\mathrm{TB}_{2}: \mathrm{PC}_{71} \mathrm{BM}\right.$ blends being less efficient), using hole-only space-charge limited-current ( $S C L C)$ devices. The blends were elaborated using identical conditions than for the solar cell devices (see the Supporting Information). In pure BET films, the mobility was found to be slightly lower than in $\mathrm{TB}_{2}$ films $\left(5 \times 10^{-3}\right.$ instead of $\left.10^{-2} \mathrm{~cm}^{2} \mathrm{~V}^{-1} \mathrm{~s}^{-1}\right)$. The dramatic drop in hole mobility (by a factor of 30 ) observed in $\mathrm{TB}_{2}: \mathrm{PC}_{61} \mathrm{BM}$ blends is considerably attenuated for BET:PC $\mathrm{C}_{71} \mathrm{BM}$, leading to a mobility of $0.8 \times 10^{-3} \mathrm{~cm}^{-2} \mathrm{~V}^{-1} \mathrm{~s}^{-1}$, which should be high enough to allow efficient charge extraction. Interestingly, the lower drop in hole mobility in BET blends occurred despite a higher fullerene content (1:1 instead of 1:0.5). These results suggest that the molecular assembly of BET is less impacted by fullerenes, possibly owing to the additional intermolecular $\pi$-stacking interactions brought in by the planar TAT units.

To further investigate the possible impact of TAT end groups on charge-carrier recombination kinetics, we analyzed the photovoltaic performances as a function of light intensity and estimated the excess carrier lifetime $\left(\tau_{\Delta n}\right)$ by transient photovoltage (TPV) and charge extraction (CE) measurements on both $B E T: P C_{71} B M$ and $\mathrm{TB}_{2}: \mathrm{PC}_{61} \mathrm{BM}$ blends (see the Supporting Information). The measurement of $\left(\tau_{\Delta n}\right)$ as a function of the chargecarrier concentration $n$ (Figure $3 \mathrm{~b}$ ) shows the existence of differing recombination mechanisms for both systems. At low values of $n$, the carrier lifetime in $\mathrm{TB}_{2}$ devices is constant, as it should be for trap-assisted recombination, whereas at high intensities, a strong decrease is observed with increasing $n$. The latter behavior hints towards bimolecular recombination, although the carrier dependence is more pronounced than usual (see the Supporting Information). In the case of BET, the $n$-independent regime was not observed in the investigated range. However, the lifetime decreases rapidly with increasing $\mathrm{n}$, while almost remaining within one order of magnitude of the values measured in $\mathrm{TB}_{2}$ devices. $\square$ ok? Defining $k_{\text {rec. }}$ as the charge-carrier recombination rate and $k_{\mathrm{ex}}$ as the charge extraction rate, the FF is expected to decrease when the ratio $k_{\text {rec. }} / k_{\text {ex. }}$ increases. ${ }^{[15]}$ The lower out-of-plane hole mobility measured by $\mathrm{SCLC}$ for $\mathrm{TB}_{2}: \mathrm{PC}_{61} \mathrm{BM}$ blends together with a lower charge recombination lifetime for this blend thus clarifies the origin of the higher FF of BET solar cells.

In summary, we designed and synthesized two new BODIPY derivatives, incorporating triazatruxene moieties as $\pi$-stacking platforms, for application in organic solar cells. The addition of a TAT platform at both ends of this dumbbell-shaped molecule was expected to increase intermolecular interconnectivity and to favor the out-of-plane hole mobility in blends. The conjugated linker between the hindered BODIPY core and the TAT platforms appears as a key parameter as only the ethynylenebased BET molecule leads to a significant photovoltaic performance improvement, with a maximum PCE of $5.8 \%$, which is the highest reported to date for a BODIPY derivative. As compared to the TAT-free reference molecule $\left(\mathrm{TB}_{2}\right)$, this increase in performance is due to a higher charge-carrier lifetime and mobility that conducts to a much higher FF in BET solar cells. Finally, we also developed a new chemical method to synthesize for the first time a 2,3,5,6-tetrastyryl BODIPY molecule with heteroaromatic substituents (BVT). Despite the lower PCE recorded for the BVT derivative, this method may greatly advance the use of unsymmetrical 2,3,5,6-tetrastyryl BODIPY molecules as candidates for organic optoelectronic applications.

\section{Acknowledgements}

This work was supported by French National Agency (ORION project ANR-13-PRGE-0001).

Keywords: chromophores - heterocycles - hydroboration photovoltaics $\cdot$ stacking interactions 
[1] G. Ulrich, R. Ziessel, A. Harriman, Angew. Chem. Int. Ed. 2008, 47, 1184 1201; Angew. Chem. 2008, 120, $1202-1219$.

[2] A. Bessette, G. S. Hanan, Chem. Soc. Rev. 2014, 43, 3342-3405.

[3] T. Bura, N. Leclerc, S. Fall, P. Lévêque, T. Heiser, P. Retailleau, S. Rihn, A. Mirloup, R. Ziessel, J. Am. Chem. Soc. 2012, 134, 17404-17407.

[4] M. C. Scharber, N. S. Sariciftci, Prog. Polym. Sci. 2013, 38, 1929-1940.

[5] O. P. Lee, A. T. Yiu, P. M. Beaujuge, C. H. Woo, T. W. Holcombe, J. E. Millstone, J. D. Douglas, M. S. Chen, J. M. J. Fréchet, Adv. Mater. 2011, 23, 5359-5363.

[6] a) I. Bulut, P. Chavez, A. Mirloup, Q. Huaulmé, B. Heinrich, A. Hébraud, S. Méry, R. Ziessel, T. Heiser, P. Lévêque, N. Leclerc, J. Mater. Chem. C 2016 4, 4296-4303; b) T. Bura, N. Leclerc, R. Bechara, P. Lévêque, T. Heiser, R. Ziessel, Adv. Energy Mater. 2013, 3, 1118-1124.

[7] H.-Y. Lin, W.-C. Huang, Y.-C. Chen, H.-H. Chou, C.-Y. Hsu, J. T. Lin, H.-W. Lin, Chem. Commun. 2012, 48, 8913-8915.

[8] Z. Xie, B. Yang, L. Liu, M. Li, D. Lin, Y. Ma, G. Cheng, S. Liu, J. Phys. Org Chem. 2005, 18, 962-973.
[9] J. Ahrens, B. Haberlag, A. Scheja, M. Tamm, M. Bröring, Chem. Eur. J. 2014, 20, $2901-2913$.

[10] L. Gai, J. Mack, H. Lu, H. Yamada, D. Kuzuhara, G. Lai, Z. Li, Z. Shen, Chem. Eur. J. 2014, 20, $1091-1102$.

[11] Z. Feng, L. Jiao, Y. Feng, C. Yu, N. Chen, Y. Wei, X. Mu, E. Hao, J. Org. Chem. 2016, 81, 6281-6291.

[12] O. Buyukcakir, O. A. Bozdemir, S. Kolemen, S. Erbas, E. U. Akkaya, Org. Lett. 2009, 11, 4644-4647.

[13] D. Zhang, Y. Wen, Y. Xiao, G. Yu, Y. Liu, X. Qian, Chem. Commun. 2008 4777-4779.

[14] Y. Tokoro, A. Nagai, Y. Chujo, Tetrahedron Lett. 2010, 51, 3451- 3454.

[15] D. Bartesaghi, I. del Carmen Pérez, J. Kniepert, S. Roland, M. Turbiez, D. Neher, L. J. A. Koster, Nat. Commun. 2015, 6, 7083.

Manuscript received: March 17, 2017

Accepted Article published: March 21, 2017

Final Article published: $\square$ U, 0000 


\section{COMMUNICATIONS}

I. Bulut, Q. Huaulmé, A. Mirloup,

P. Chávez, S. Fall, A. Hébraud, S. Méry,

B. Heinrich, T. Heiser, P. Lévêque, ${ }^{*}$

N. Leclerc*

$\mathbf{a - n}$

Rational Engineering of BODIPYBridged Trisindole Derivatives for Solar Cell Applications

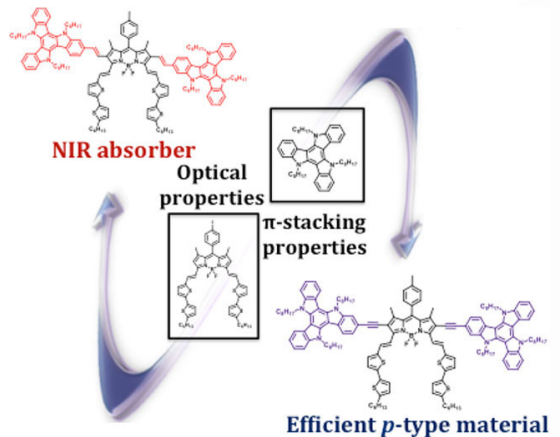

Let's get ready to dumbbell! Boron dipyrromethene-based (BODIPY) dumbbell-shaped molecules, including a first 2,3,5,6-tetravinyl aromatic BODIPY derivative, have been synthesized to strengthen $\pi$-stacking abilities. Used as a p-type material, high fill factors can be obtained in bulk heterojunction solar cells when blended with a fullerene derivative, leading to a new BODIPY-based record power conversion efficiency of $5.8 \%$.

Share your work on social media! ChemSusChem has added Twitter as a means to promote your article. Twitter is an online microblogging service that enables its users to send and read text-based messages of up to 140 characters, known as "tweets". Please check the pre-written tweet in the galley proofs for accuracy. Should you or your institute have a Twitter account, please let us know the appropriate username (i.e., @accountname), and we will do our best to include this information in the tweet. This tweet will be posted to the journal's Twitter account @ChemSusChem (follow us!) upon online publication of your article, and we recommended you to repost ("retweet") it to alert other researchers about your publication.

Please check that the ORCID identifiers listed below are correct. We encourage all authors to provide an ORCID identifier for each coauthor. ORCID is a registry that provides researchers with a unique digital identifier. Some funding agencies recommend or even require the inclusion of ORCID IDs in all published articles, and authors should consult their funding agency guidelines for details. Registration is easy and free; for further information, see http://orcid.org/.

Dr. Ibrahim Bulut

Dr. Quentin Huaulmé

Dr. Antoine Mirloup

Dr. Patricia Chávez

Dr. Sadiara Fall

Dr. Anne Hébraud

Dr. Stéphane Méry

Benoît Heinrich

Prof. Thomas Heiser

Dr. Patrick Lévêque

Dr. Nicolas Leclerc 